\title{
The Perils of Using Mechanical Turk to Evaluate Open-Ended Text Generation
}

\author{
Marzena Karpinska Nader Akoury Mohit Iyyer \\ University of Massachusetts Amherst \\ \{mkarpinska, nsa, miyyer\} @cs.umass.edu
}

\begin{abstract}
Recent text generation research has increasingly focused on open-ended domains such as story and poetry generation. Because models built for such tasks are difficult to evaluate automatically, most researchers in the space justify their modeling choices by collecting crowdsourced human judgments of text quality (e.g., Likert scores of coherence or grammaticality) from Amazon Mechanical Turk (AMT). In this paper, we first conduct a survey of 45 open-ended text generation papers and find that the vast majority of them fail to report crucial details about their AMT tasks, hindering reproducibility. We then run a series of story evaluation experiments with both AMT workers and English teachers and discover that even with strict qualification filters, AMT workers (unlike teachers) fail to distinguish between model-generated text and human-generated references. We show that AMT worker judgments improve when they are shown model-generated output alongside human-generated references, which enables the workers to better calibrate their ratings. Finally, interviews with the English teachers provide deeper insights into the challenges of the evaluation process, particularly when rating model-generated text.
\end{abstract}

\section{Introduction}

Recent advances in neural language modeling have spurred research into open-ended text generation tasks such as story generation (Peng et al., 2018a), style transfer (Krishna et al., 2020), and pun generation (He et al., 2019). Since the space of possible outputs for these tasks is huge compared to more constrained problems such as machine translation, automatic metrics such as BLEU (Papineni et al., 2002) and ROUGE (Lin, 2004) that measure similarity to reference texts are mostly uninformative (Akoury et al., 2020). ${ }^{1}$ Human evaluation of

\footnotetext{
${ }^{1}$ Nevertheless, such metrics are commonly reported in research papers on open-ended text generation.
}

model-generated text, which is critical for openended tasks given the unreliability of automatic metrics (Peng et al., 2017; Reiter, 2018; See et al., 2019), is frequently conducted on Amazon's popular Mechanical Turk platform (AMT) to minimize cost and time. Most existing AMT studies ask crowdworkers to provide Likert scale ratings of various properties of generated text, such as $f l u$ ency and likability.

In this paper, we study the reliability and reproducibility of AMT evaluations of open-ended text generation. We first conduct a survey of papers on open-ended text generation between 2018-2020 and find many critical details often go unreported (e.g., worker qualifications, payment, task descriptions, annotator agreement), a finding in line with prior reproducibility studies outside open-ended text generation (Card et al., 2020; Howcroft et al., 2020; van der Lee et al., 2021).

Next, we perform a series of story generation evaluations with both AMT workers and expert raters (English teachers), applying a variant of the most common task configuration that appeared in our survey (5 point Likert scale ratings of 200 examples with three annotators per example) to the paragraph-length WritingPrompts dataset of Fan et al. (2018). Unlike prior work in this area, we ask raters to evaluate both stories generated by a fine-tuned GPT-2 language model (Radford et al., 2019) and human-written reference stories on the same scale, as we expect the latter to consistently score higher on all evaluations. Our experiments expose and quantify several troubling trends:

1. AMT ratings do not reliably distinguish model-generated text from human-generated text unless workers are asked to rate both sideby-side, which allows them to better calibrate their ratings.

2. Running an identical task (same AMT parameters and input data) on different days of the 
(a) While teachers unanimously marked this story as irrelevant to the prompt, an AMT worker spent $\sim 19 \mathrm{sec}$ reading it before rating it a 5 out of 5 on relevance.

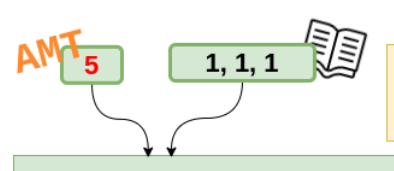

Prompt: The Aliens want to know what the hell we did with Atlantis. GPT-2

It was a small village nestled in the mountains of Colombia. There was a little village here. All of it belonged to a little girl named Kate. She was a greenhorn by the name of Jorge, but he wasn't surprised that he, and a woman named Joan were the ones making the efforts to get her to a healer. And, of course, they had provided her with healthy water, safe food, and medical care. (...) (b) Well written story was rated as 1 out of 5 for grammar by a worker on AMT.

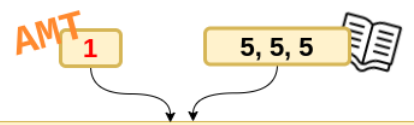

Prompt: The taboo over necromancy is a thing of the past. You live in a world where it is normal to become a lich and "live" forever. HUMAN REFERENCE

"You're getting old."

It was a gray and miserable afternoon but Jill didn't really seem to notice. Her cappuccino caught raindrops.

"I know, I know," dismissed Max. His latte had a lid. The two sipped their coffee in silence. Only one of them noticed the barista added way too much caramel.

"I..." began Jill. She fidgeted with her mug. “'I'm scared of losing you." (...) (c) Gaps in world knowledge affected ratings of English teachers. A teacher unfamiliar with the show "The Office" rated the story lower for coherence.

Prompt: The Office's Michael Scott has gotten
wind of the show Undercover Boss and decides he
wants to go undercover at Dunder-Mifflin Scranton.
HUMAN REFERENCE
MICHAEL has his back to the wall that leads to the
OPEN WAREHOUSE GARAGE DOOR. He's trying to
stand like he's being very stealthy, palms against the
wall.
MICHAEL: I Just finished Season 1 of 'Undercover
Boss" on DVD... well it was only the first disc of the
first season. (...)

Figure 1: Three examples of prompt-story pairs along with ratings from AMT workers and expert teachers that demonstrate major issues with human evaluation of open-ended text generation.

week exhibits high variance and can lead to dubious conclusions (e.g., that reference texts are lower quality than GPT-2 generated text).

3. Many AMT workers do not carefully read the text that they are evaluating. Even after enabling multiple qualifications to exclude lowquality workers, $42 \%$ of workers on average take fewer than 40 seconds to complete each task. Filtering out these workers can make a significant impact to the overall ratings, but also notably reduces the number of datapoints.

4. Even expert raters struggle to read and judge model-generated text. The time they spend per example increases significantly compared to that for references, and agreement also drops.

For future human evaluations of open-ended text generation tasks, we urge researchers to obtain expert raters whenever possible. If AMT is the only feasible option, we recommend that available reference outputs also be evaluated alongside modelgenerated ones to improve rating calibration, and also that heavy filtering of the worker population (possibly through qualification tasks, or post-hoc removal) is performed prior to reporting results.

\section{A survey of papers that evaluate open-ended text generation with AMT}

We begin with a survey of 45 papers that use AMT to evaluate the output of open-ended Englishlanguage text generation models, which includes generated stories, metaphors, paraphrases, puns, sarcasm, and sentences with transferred style or attributes ${ }^{2}$. Each paper was published between 2018 and 2020 at ACL, NAACL, or EMNLP, and we exclude papers that use AMT to evaluate more wellestablished generation tasks like machine translation, or summarization. ${ }^{3}$ Unlike previous surveys of evaluating generated text (Çelikyilmaz et al., 2020; van der Lee et al., 2021), we focus specifically on AMT evaluations of open-ended text generation. In this section, we provide an overview of the different types of evaluation task setups present in our survey; later, we experiment with several variants of the most common setup.

Evaluation criteria: As in the survey of Howcroft et al. (2020), we observe a variety of different evaluation criteria and definitions of these criteria across the 45 papers. The most common criteria include fluency and/or grammaticality (19), overall "quality" (12), and relevance (10) to a corresponding prompt. Furthermore, stories in particular tend to also be evaluated on some notion of coherence (9) and likability (4).

\footnotetext{
${ }^{2}$ The details on the survey questions and surveyed papers are provided in the Appendix A

${ }^{3}$ For detailed numbers see Table A1; the specific papers in the survey are in Table A2. Four papers conducted multiple evaluations with different settings, and as such we count them in multiple categories. Moreover, all but nine papers evaluated their systems on more than one attribute.
} 
Rating scales: More than half of the papers (24) employ a 5-point Likert scale to evaluate the above criteria; of these, 19 provided labels for just the end points of the scale (e.g., "lowest" vs. "highest"), while 5 labeled all points on the scale. The next most common evaluation type is ranking two or more system outputs (23). Less common are other Likert scales (3, 4, or 6-point), pass/fail tasks, and output-prompt matching tasks.

Number of raters and rated items: An alarming number of papers (14) do not even report the number of raters and/or items (6) used for evaluation. Of the remaining, most papers (16) obtain ratings from 3 separate AMT workers per item. The most common number of items per evaluation is 100 (14). The number of raters per item in other studies ranges from 2 to 11 , while the number of items ranges from 12 to 1,000 .

Workers qualifications and compensation: The vast majority of papers do not report AMT worker qualifications (32) or worker compensation (35), which adds to the reproducibility woes. Among papers that report qualifications, the most common were HIT $^{4}$ approval rate $\geq 90 \%-99 \%$, and number of approved HITs between 500 to 5,000 . Only 11 papers mentioned restricting workers to those from English-speaking countries or applying some kind of language test, despite all evaluations being done on English text.

Length of the Rated Text: As open-ended text generation encompasses an array of different tasks, the length of the rated text differed greatly, ranging from single sentences (28), sometimes presented in a longer context, to short paragraphs (7), and longer paragraphs (14). The latter setting is most commonly used for story generation tasks.

\section{Evaluating story generation with AMT}

Our survey reveals that the most popular Mechanical Turk task design for open-ended text generation asks AMT workers to rate various properties of generated text on a 5-point Likert scale. In this section, we conduct a series of AMT evaluations for the open-ended problem of story generation by varying different parameters within this standard task design. Importantly, we evaluate both modelgenerated stories as well as human-generated refer-

\footnotetext{
${ }^{4}$ In AMT parlance, a human intelligence task (HIT) refers to a single item; in our case, each HIT corresponds to one story, which workers rate on four different properties.
}

ence stories, which provides a pseudo upper bound for the ratings. Our experiments reveal that worker qualifications (e.g., HIT approval rate and number of accepted HITs) do not notably impact judgments or spam rate on reference stories, with the exception of country of origin. Furthermore, we uncover an issue with rating calibration: when both reference and model-generated stories are included for the same prompt, average reference scores are significantly higher than those for model-generated text; however, when workers only see one type of text per HIT, they give similar average scores to both types.

\subsection{Experimental Setup}

We first describe the parameters of our experiments before later analyzing the results.

Dataset: We use the WritingPrompts dataset collected by Fan et al. (2018), which is a collection of 303,358 English language stories written by Reddit users on the r/WritingPrompts subreddit..$^{5}$ This dataset, which consists of short prompts paired with user-written stories (e.g., "There are 10 legendary dentists who review every toothpaste. You are the 10th... being hunted by the other 9..."), has been used in multiple previous works on paragraph-length story generation (Fan et al., 2019; See et al., 2019; Mao et al., 2019). We randomly select 200 prompts from the test set for all of our experiments. Since the human-written stories in the dataset are already tokenized, we first de-tokenized the stories, cleaned up artifacts from lemmatization, and manually truncated each story so that it ends with a full sentence and is no longer than 150 words in order to make the length comparable with the machine-generated story. ${ }^{6}$ We use the resulting stories for all experiments with reference text.

Model-generated stories: We follow a similar modeling approach to prior story generation work (Mao et al., 2019; Guan et al., 2020) by fine-tuning a pretrained GPT-2 medium-sized model (Radford et al., 2019) on the training set of the WritingPrompts dataset, using the HuggingFace Transformers library (Wolf et al., 2020). We use a batch size of approximately $50 k$ tokens, a learning rate of $5 e-5$ with a linear learning rate schedule, and train for 3 epochs, stopping training

\footnotetext{
${ }^{5}$ https: //reddit.com/r/WritingPrompts /

${ }^{6}$ The mean length of the selected reference stories is 134.5 tokens, with a standard deviation of 8.81 .
} 
after validation perplexity converges to $\sim 19$. Each training example consists of a concatenation of a prompt, separator token (new line character), and reference story. At test-time, we feed the same 200 prompts selected above to our model for fair comparison to the human-written stories, and we generate three stories per prompt using nucleus sampling (Holtzman et al., 2019) with $p=0.9$. We manually truncate each sample so that it ends with a full sentence and is no longer than 150 words. $^{7}$ These stories are used in all experiments evaluating machine-generated stories.

AMT task parameters: We conduct all experiments using the default interface in Mechanical Turk (see Figure A1 and Figure A2). Workers were asked to rate human-written and/or machinegenerated stories on four attributes, with the following definitions provided to them:

1. Grammar: "How grammatically correct is the text of the story fragment?"

2. Coherence: "How well do the sentences in the story fragment fit together?"

3. Likability: "How enjoyable do you find the story fragment?"

4. Relevance: "How relevant is the story fragment to the prompt?"

Their ratings fall on a 5-point Likert scale with the corresponding endpoints labelled as "lowest" (1 point) and "highest" (5 points). Since our survey did not find many previous papers that reported using detailed descriptions for each point on the scale, we chose to use minimal labels to mimic the most popular setup (see Section 2 for details).

Each of our AMT experiments shows workers the same 200 prompts paired with human and/or machine-generated stories, and we solicit three worker judgments per HIT. Workers were paid $\$ 0.20$ per HIT for tasks that showed one story, and $\$ 0.35$ per HIT for those that showed two stories; in total, our AMT experiments cost roughly $\$ 1.5 \mathrm{~K}$. Importantly, each experiment used a completely different set of workers (i.e., each worker could only participate in one experiment, although they can complete multiple HITs within that experiment), which is an intentional choice to prevent workers from judging the same story multiple times. Finally, to eliminate potential variations stemming

\footnotetext{
${ }^{7}$ The mean length of the generated stories is 137.4 tokens with a standard deviation of 8.36 .
}

from evaluation on different days (weekdays vs. weekends) and time of day, we launch all experiments on weekdays between 11:00-11:30AM PST.

\subsection{AMT Evaluations of Reference Text}

Our first set of experiments concerns only humanwritten reference stories; we move to machinegenerated text in the next subsection. One of our assumptions with human-written stories, supported by the expert teacher assessment in Section 4, is that they should receive relatively high scores for all four properties (except perhaps likability which is highly subjective). We thus use reference texts to evaluate various AMT parameters such as qualifications or day of task launch, observing how modifications to these parameters affect the average scores of reference text.

Impact of worker qualifications: We run four experiments evaluating the previously-described set of 200 prompts with reference story fragments, varying the worker qualifications as follows: (1) no qualifications, (2) including only workers with HIT approval rate $>90 \%$, (3) including only workers with approval rate $>90 \%$ and at least 1000 approved HITs, (4) including only workers with approval rate $>90 \%$ and at least 1000 approved HITs who are located in English-speaking countries. ${ }^{8}$

The results in the top portion of Table 1 suggest that applying all of the qualifications (i.e., workers from English-speaking countries, approval rate > $90 \%$, approved HITs $\geq 1000$ ) has a positive effect on the quality of workers, as this setting yielded the highest scores out of the four experiments for $\mathrm{Co}^{-}$ herence and relevance while ratings for grammar were also considerably high. Ratings for likability were lower than in the experiments with less strict qualifications, but likability is a very subjective measure which consistently shows a very low agreement (Krippendorff's $\alpha$ of -0.04 to 0.11 ). When all AMT worker qualifications are enabled, the worker ratings more closely align to those made by English teachers, although there are still substantial deviations (Section 4). Additionally, with all qualifications enabled, workers show higher agreement for grammar, coherence, relevance and even likability, although the agreement between raters remains low.

High variance across different days: Concerned by the low overall agreement, we decided

\footnotetext{
${ }^{8}$ We include workers from the US, Canada, the UK, Australia, and New Zealand.
} 


\begin{tabular}{|c|c|c|c|c|c|c|c|c|}
\hline \multirow{2}{*}{ Experiment description } & \multicolumn{2}{|c|}{ Grammar } & \multicolumn{2}{|c|}{ Coherence } & \multicolumn{2}{|c|}{ Relevance } & \multicolumn{2}{|c|}{ Likability } \\
\hline & Mean $_{S T D}$ & $\overline{\mathrm{IAA}} \%$ & Mean $_{S T D}$ & IAA $\%$ & Mean $_{S T D}$ & IAA $\%$ & $\mathrm{Mean}_{S T D}$ & $\mathrm{IAA} \%$ \\
\hline & \multicolumn{8}{|c|}{ Impact of Qualifications } \\
\hline No qualifications & $4.05_{0.90}$ & $0.08_{14.5}$ & $3.92_{0.98}$ & $0.02_{4.5}$ & $3.66_{1.22}$ & $0.13_{11}$ & $3.64_{1.16}$ & $0.02_{7}$ \\
\hline$+>90 \%$ HIT approval & $4.16_{0.86}$ & $0.07_{18}$ & $4.07_{0.93}$ & $0.06_{10.5}$ & $3.67_{1.14}$ & 0.079 & $3.68_{1.15}$ & $0.08_{10}$ \\
\hline + at least 1000 HITs & $3.91_{0.85}$ & $0.05_{12}$ & $3.85_{0.98}$ & $0.08_{11.5}$ & $3.60_{1.15}$ & $0.18_{8}$ & 3.631 .13 & $0.07_{12.5}$ \\
\hline \multirow[t]{2}{*}{ + English-speaking countries } & $4.00_{0.92}$ & $0.21_{15.5}$ & $4.11_{0.96}$ & $0.14_{16.5}$ & $3.71_{1.26}$ & $0.27_{10}$ & $3.37_{1.18}$ & $0.11_{7.5}$ \\
\hline & \multicolumn{8}{|c|}{ Variance Across Days } \\
\hline Day 1 (all quals.) & $4.00_{0.92}$ & $0.21_{15.5}$ & $4.11_{0.96}$ & $0.14_{16.5}$ & $3.71_{1.26}$ & $0.27_{10}$ & $3.37_{1.18}$ & $0.11_{7.5}$ \\
\hline Day 2 (all quals.) & $3.86_{0.92}$ & $-0.03_{10.5}$ & 3.920 .98 & $-0.03_{6.5}$ & $3.71_{1.08}$ & $0.02_{11}$ & $3.73_{0.97}$ & $-0.04_{8.5}$ \\
\hline \multirow[t]{2}{*}{ Day 3 (all quals.) } & $3.98_{0.96}$ & $0.18_{11}$ & $4.05_{0.94}$ & $0.13_{10.5}$ & $3.46_{1.29}$ & $0.26_{8}$ & $3.42_{1.16}$ & $0.07_{4.5}$ \\
\hline & \multicolumn{8}{|c|}{ Impact of Country of Origin } \\
\hline \multirow[t]{2}{*}{ - English-speaking countries } & $3.82_{1.04}$ & $0.03_{11}$ & $3.45_{1.19}$ & $-0.01_{9}$ & $3.25_{1.27}$ & $0.03_{6.5}$ & $3.32_{1.26}$ & $-0.09_{3}$ \\
\hline & \multicolumn{8}{|c|}{ Impact of Filtering by the Median Work Time } \\
\hline Day $2($ Median $\geq 40 \mathrm{~s})$ & $4.04_{0.94}$ & & $4.33_{0.92}$ & & $3.74_{1.34}$ & & $3.67_{1.06}$ & \\
\hline
\end{tabular}

Table 1: AMT experiments on human-written reference stories. Inter-annotator agreement (IAA) between the three raters is measured with Krippendorff's $\alpha$ as well as the percentage of stories for which all three raters exactly agreed on a rating (the latter is subscripted). Last section omits IAA due to the large number of missing datapoints. Statistical significance for the relations between groups is provided in Appendix D.

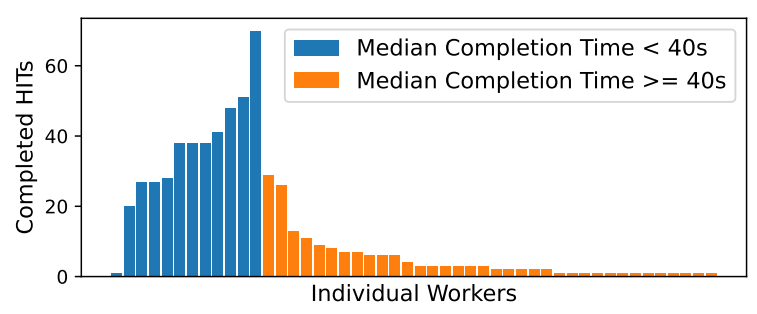

Figure 2: This example from Day 2 shows that many AMT workers complete multiple HITs in less time and with lower agreement in comparison to our experts.

to run another set of experiments that repeats the same experiment (all qualifications enabled) across three different days. Due to our constraint that each worker can only participate in one experiment, each of these experiments has a different subset of qualified workers. As shown in the second portion of Table 1, although the first and third days yielded similar mean ratings/agreement in terms of grammar $(\mathrm{M}=4.00, \mathrm{IAA}=0.21 \mathrm{vs} \mathrm{M}=3.98$, IAA $=0.18)$ and coherence $(\mathrm{M}=4.11, \mathrm{IAA}=0.14 \mathrm{vs}$ $\mathrm{M}=4.05, \mathrm{IAA}=0.13$ ), the second day received lower ratings across the board and had overall poor IAA (see Table 1). Furthermore, ratings for relevance in the third day $(\mathrm{M}=3.46)$ were significantly lower than in the first two days $(M=3.71)$, which indicates that simply using all AMT qualifications is not enough to achieve consistent results.

\section{Many AMT workers do not spend enough time} reading the stories: The low overall agreement also motivated us to examine the average time each worker spent per HIT. While AMT reports WorkTimeInSeconds in the results file made available to task requesters, we observe similar to Akoury et al. (2020) that these times are artificially inflated due to workers who accept multiple HITs at the same time and work on them sequentially (e.g., in different tabs). Such workers are also frequently among the most prolific in terms of HITs completed per experiment (see Figure 2), since there is no maximum number of HITs per worker. ${ }^{9}$ We correct for this by measuring the time between consecutively submitted HITs by the same worker, which can be derived by analyzing start and end times of each HIT. This "actual time" differs considerably from the AMT reported WorkTimeInSeconds: for instance, a worker that AMT reports had a mean work-time of 360 seconds had an actual mean working time ${ }^{10}$ of $22 \mathrm{~s}$ and a median of $13 \mathrm{~s}$. To put these numbers in perspective, this is about one-fourth of the time that the fastest English teacher achieved (see Section 4).

As it is impossible to carefully read a paragraphlength story and assess all four properties in as little as 13 seconds, we measure the impact on average

\footnotetext{
${ }^{9}$ Like most AMT tasks (Fort et al., 2011; Robinson et al., 2019), the majority of HITs for our evaluations are provided by a small fraction of workers. The majority of workers provided ratings for only one or two stories while a very few productive workers rated over $50 \%$ of the stories (see Figure 2).

${ }^{10}$ The mean work time is also not very representative as workers typically accept multiple HITs, wait a period of time, then submit all accepted HITs in quick succession.
} 
ratings when filtering out workers who spend too little time per HIT (last row of Table 1). Specifically, we remove judgments from workers whose median time is below 40s (which is a low bar), and find that on average about $42 \%$ of our ratings are filtered out (ranging from $20 \%-72 \%$ across all experiments). ${ }^{11}$ Of our surveyed papers, only Akoury et al. (2020) report actual work time, demonstrating that this is a major issue in modern AMT evaluations of text quality that most researchers have overlooked.

Impact of worker country of origin: While all of the surveyed papers evaluate only English text, only 11 of them reported using some kind of filtering to ensure that workers have sufficient knowledge of English. The default AMT setting does not filter workers by country of origin, which potentially increases the variance of results depending on the English proficiency of workers who accept HITs. To measure this, we re-run our experiment with all qualifications, except we restrict the task to only workers from countries that do not primarily speak English (i.e., we exclude workers from the US, Canada, UK, Australia, New Zealand, Ireland, and Singapore). The third portion of Table 1 shows that workers from non-English speaking countries rated coherence, relevance, and grammar $^{12}$ significantly lower than identically-qualified workers from English-speaking countries (Day 13). Thus, researchers rating English text should restrict their tasks to English-speaking countries, although Kennedy et al. (2020) find that many workers use Virtual Private Networks (VPNs) to take part in tasks restricted to those in the US.

\subsection{Evaluating Machine-Generated Text}

We now turn to AMT evaluation of machinegenerated stories produced by the GPT- 2 model described in subsection 3.1. Based on our previous experiments with reference texts, we select the "all qualifications" setting (i.e., workers from Englishspeaking countries, approval rate $>90 \%$, approved HITs $\geq 1000$ ) for all GPT-2 AMT tasks. We study

\footnotetext{
${ }^{11} \mathrm{We}$ also ran experiments with even stricter qualification filters (i.e., acceptance rate $\geq 99 \%$ and at least 10,000 approved tasks), but this made no notable difference to the percentage of data being filtered out (35\%). This is most likely due to the fact that most requesters are reluctant to reject HITs regardless of quality, which results in an estimated $95 \%$ of workers having an approval rate of $98 \%$ or above (Matherly, 2019; Wessling et al., 2017).

${ }^{12}$ There was no significant difference between grammar ratings collected from raters from non-English speaking countries and ratings collected on Day 2.
}

two different conditions: (1) HITs contain a prompt and a GPT-2 generated text, and (2) HITs contain a prompt and both a human-written reference story as well as a GPT-2 generated story. In the latter case, we ask AMT workers to rate both texts on each of the four properties. Overall, we observe that workers cannot effectively distinguish between reference and model-generated stories when they are evaluated separately (in terms of average ratings), but that this distinction emerges clearly when they are presented with both types of stories in the same HIT.

When presented only GPT-2 generated text, AMT worker ratings rate them similarly to reference texts, despite obviously worse quality: In our first experiment, we follow the protocol from our experiments with human-written reference stories, showing AMT workers a prompt and a model-generated story and asking them to rate it on the same attributes (grammar, coherence, relevance, and likability). The results of this evaluation are presented in the upper row of Table 2 along with the three sets of ratings of reference stories obtained with the same "all qualifications" setting from before (Days 1-3 in Table 1).

Surprisingly, GPT-2 output is not consistently rated significantly lower than human-written text. For instance, workers in Day 2 rated human-written stories similarly to the GPT-2 generated stories in terms of grammar $(\mathrm{M}=3.86$ vs. $\mathrm{M}=3.94)$ and coherence $(\mathrm{M}=3.92$ vs. $\mathrm{M}=3.82)$, while workers in Day 3 rated human-written stories as similarly relevant to the prompt as GPT-2 output ( $\mathrm{M}=3.46$ vs. $M=3.44)$. Depending on which reference day we compare the GPT-2 output to, GPT-2 is rated similarly to human-written stories in terms of all four properties, which indicates that this evaluation is uninformative; nevertheless, the majority of surveyed papers use exactly this task design to obtain ratings for model-generated output.

Asking workers to rate both human-written and model-generated stories side-by-side improves ratings: We hypothesize that the previous result is due to scale calibration differences between the two settings: when repeatedly confronted with incoherent model-generated text, a worker may be more generous with their ratings compared to if they only see coherent human-written text. Thus, we explore whether their ratings can be better calibrated by asking them to rate both types of 


\begin{tabular}{|c|c|c|c|c|c|c|c|c|c|}
\hline \multirow[t]{2}{*}{ Raters } & \multirow[t]{2}{*}{ Type of text } & \multicolumn{2}{|c|}{ Grammar } & \multicolumn{2}{|c|}{ Coherence } & \multicolumn{2}{|c|}{ Relevance } & \multicolumn{2}{|c|}{ Likability } \\
\hline & & $\operatorname{Mean}_{S T D}$ & $\mathrm{IAA} \%$ & $\operatorname{Mean}_{S T D}$ & $\mathrm{IAA} \%$ & Mean $_{S T D}$ & $\mathrm{IAA} \%$ & Mean $_{S T D}$ & IAA $\%$ \\
\hline & \multicolumn{9}{|c|}{ AMT workers fail to effectively distinguish between human written and GPT-2 generated stories } \\
\hline AMT & f. (Day 1) & $4.00_{0.92}$ & $0.21_{15.5}$ & $4.11_{0.96}$ & $0.14_{16.5}$ & $3.71_{1.26}$ & $0.27_{10}$ & $3.37_{1.18}$ & 0.1 \\
\hline AMT & & $3.86_{0.92}$ & -0.0 & 0.98 & -0.0 & 1.08 & & $3.73_{0.97}$ & $-0.04_{8.5}$ \\
\hline AMT & & $3.98_{0.96}$ & 0.1 & & & & & $3.42_{1.16}$ & \\
\hline \multirow[t]{2}{*}{ AMT } & GPT-2 & $3.94_{0.93}$ & $0.11_{17.5}$ & $3.82_{1.12}$ & $0.05_{7.5}$ & $3.44_{1.41}$ & $0.10_{7}$ & $3.42_{1.25}$ & $0.02_{4.5}$ \\
\hline & \multicolumn{9}{|c|}{$A M T$ workers score GPT-2 lower when also presented with reference text } \\
\hline AMT & Refe & $3.83_{0.99}$ & & $3.83_{1.1}$ & & $3.49_{1.26}$ & & 3.4 & $0.03_{6.5}$ \\
\hline \multirow[t]{2}{*}{ AMT } & GPT-2 & $3.82_{0.90}$ & $0.10_{12}$ & $3.39_{1.1}$ & $0.04_{9.5}$ & $2.70_{1.26}$ & $0.06_{6.5}$ & 2.991 .14 & $-0.04_{4}$ \\
\hline & \multicolumn{9}{|c|}{ Teachers rate GPT-2 generated stories lower than AMT workers } \\
\hline Teachers & Reference & $4.50_{0.83}$ & $0.19_{35.5}$ & & & 3.821 .38 & & $3.69_{1.30}$ & $-0.01_{5}$ \\
\hline Teachers & GPT-2 & $4.56_{0.62}$ & $0.00_{24.5}$ & $3.73_{1.19}$ & $0.17_{13}$ & $2.54_{1.49}$ & $0.54_{25.5}$ & $2.96_{1.46}$ & $-0.07_{3}$ \\
\hline
\end{tabular}

Table 2: Comparison of AMT workers and expert teachers on both human and machine-generated text. Interannotator agreement (IAA) between the three raters is measured with Krippendorff's $\alpha$ as well as the percentage of stories for which all three raters exactly agreed on a rating (the latter is subscripted). Statistical significance for the relations between groups is provided in Appendix C and Appendix D.

stories side-by-side, using the same qualification settings as for the other experiments. The results of this experiment are presented in the middle row of Table 2. Workers score GPT-2 generated stories significantly lower than reference stories on coherence $(\mathrm{M}=3.39$ vs. $\mathrm{M}=3.83)$, relevance $(\mathrm{M}=2.70$ vs. $\mathrm{M}=3.49)$, and likability ( $\mathrm{M}=2.99$ vs. $\mathrm{M}=3.48)$, which is in line with our expectations. Their ratings for grammar $(\mathrm{M}=3.82$ vs. $\mathrm{M}=3.83)$ are similar for both types of text, which we also observe with expert teacher ratings in Section 4 and is expected since GPT-2's output is generally grammatical.

\section{Evaluation by expert teachers}

The experiments in the previous section demonstrate the unreliability of AMT ratings for openended text generation, even when qualifications are used to restrict the task to ostensibly reliable workers. In this section, we compare the ratings produced by AMT workers to those of expert raters, specifically a set of three English teachers, and discover significant deviations between the two groups. Though they rated both types of stories separately, their ratings clearly distinguish between human-written references and machine-generated stories. We also conducted post-task interviews with the teachers and organized a mediation session to discuss stories with high disagreement, observing that they reach consensus after discussion in about $80 \%$ of cases.

Recruiting English teachers: We choose English teachers as experts for our story generation task because they regularly evaluate student-written papers and are experienced at detecting both lowlevel grammatical mistakes as well as discourselevel issues with logical coherence. The three teachers were recruited from the authors' personal networks, and each of them either has a degree in teaching English as a Second Language or a CELTA certificate. ${ }^{13}$ They were paid $\$ 125$ each for participating in our experiments, which required them to rate the same 200 human-written stories and 200 GPT-2 generated stories on the same four properties as that of the AMT workers, given an identical task interface. ${ }^{14}$

Unlike AMT workers, teachers rate reference stories higher than GPT-2 generated ones: We asked teachers to first rate the 200 reference stories, and then a week later to rate the GPT- 2 generated stories. Just like the AMT workers, they were not told that the text in the second task was machinegenerated. Importantly, we used the same set of teachers for both tasks, so they already had significant experience with the task when rating the machine-generated text (as opposed to using new AMT workers for each experiment).

The results of this evaluation are presented in the last row of Table 2. Unsurprisingly, teachers rated human-written stories significantly higher than GPT-2 generated stories in terms of coherence $(\mathrm{M}=4.38$ vs. $\mathrm{M}=3.73)$, relevance $(\mathrm{M}=3.82$ vs. $\mathrm{M}=2.54)$, and likability ( $\mathrm{M}=3.69$ vs. $\mathrm{M}=2.96)$ (all

\footnotetext{
${ }^{13}$ Certificate in Teaching English to Speakers of Other Languages.

${ }^{14}$ The teachers completed their ratings using an identical version of our task deployed on the AMT sandbox environment.
} 
$p$ 's $<0.001)$. On the other hand, they rated humanwritten stories and GPT-2 generated stories as similar in terms of grammar ( $\mathrm{M}=4.50$ vs. $\mathrm{M}=4.56$ ). Moreover, teachers' ratings of human-written stories are considerably higher than AMT ratings for all attributes except likability $(\mathrm{M}=3.69)$ which depending on the day was rated lower $\left(\mathrm{M}_{\text {Day } 1}=3.37\right)$ or higher $\left(\mathrm{M}_{\text {Day2 }}=3.73\right)$ by the AMT workers. Similarly, teachers' ratings of GPT-2 stories are lower than the ratings we obtained from AMT workers for coherence ( $\mathrm{M}=3.73$ vs. $\mathrm{M}=4.11)$, relevance $(\mathrm{M}=2.54$ vs. $\mathrm{M}=3.71)$, and likability $(\mathrm{M}=2.96$ vs. $\mathrm{M}=3.37)$.

Teachers need to see many examples to properly calibrate their ratings: In post-task interviews, all teachers reported that it took them 10-20 stories on average to calibrate their ratings. Since most AMT workers complete only one to two HITs, they do not have similar time to get acquainted with the task; this may suggest that having a pre-task training phase can improve worker calibration.

Coherence is difficult to rate for machinegenerated text: The teachers unanimously report that while coherence is easy to rate for reference stories (since most of them are largely coherent), it is the most difficult property to rate for GPT-2 generated stories. Since they did not know that they were rating machine-generated text, they spent time trying to make sense of the author's possible intent in producing many of the strange artifacts and hallucinations common to output of neural language models (Holtzman et al., 2019). In contrast, relevance turned out to be the easiest property of machine-generated text for teachers to rate, which is expected as many of GPT-2's stories deviate very quickly from the prompt (see Figure 1).

GPT-2 generated stories are much harder for teachers to rate overall: All teachers reported struggling more when rating GPT-2 stories, a fact reflected in their average rating time per story increasing significantly from 69.8 seconds to 87.3 seconds $(p<0.05)$. In contrast, the average rating time of AMT workers decreased from $135.3 \mathrm{sec}-$ onds for human-written text (Day 1) to $91.5 \mathrm{sec}-$ onds for GPT- 2 text $(p<0.05)^{15}$. Teachers also reported having to recalibrate their scale when rating

\footnotetext{
${ }^{15}$ This time was computed by the researchers to account for workers accepting multiple HITs at the same time, however, the WorkTimeInSeconds reported in the AMT results shows similar trends.
}

the GPT-2 generated stories, as the stories were significantly worse than the human-written text. Consequently, they suggested that it would be easier to calibrate their scale had the GPT- 2 output been presented beside the human-written text, which supports the results from our joint rating task with AMT workers. Finally, the teachers suggested that creating a standardized rubric would greatly facilitate the rating process. This step is even more important as machine-generated text faces different issues than human-written text.

Resolving teacher disagreement: One advantage of using human expert raters is that we can easily have them discuss examples on which they disagree. We arranged a mediation meeting between two of the three teachers to discuss 60 stories on which they showed the highest disagreement (3 attributes $\times 10$ stories $\times 2$ types, we excluded likability due to its subjective nature). In this meeting, they were first asked to rate the stories again, without being provided their previous rating. In about $20 \%$ of cases, one of the teachers disagreed with their own previous rating due to honest lapses of judgment. Another common reason for disagreement was missing world knowledge (see Figure 1, right). One more reason for disagreement, a confusion about how to rate slang in terms of grammaticality. While the text was not correct in the view of the official grammar, it was appropriate for the prompt, so one teacher rated it high while the other rated it low. Overall, after discussing examples that they still disagreed on after re-rating, teachers were able to come to a consensus on $80 \%$ of the stories; the remaining disagreements persisted due to individual differences in strictness. See Appendix C for details on the mediation meeting.

Replicating the study on Upwork: We recognize that replicating our study is difficult without access to a network of English teachers. As such, we performed the same experiment using three certified teachers recruited on a freelance platform, Upwork. ${ }^{16}$ The teachers were paid $\$ 175$ for evaluating the same 200 human-written and 200 GPT-2 generated stories using the exact same setup as in subsection 3.1. It took approximately one week to collect the data (including break between rating human-written and GPT-2 generated stories). The results obtained via Upwork were comparable with the results obtained from the English teachers de-

\footnotetext{
${ }^{16}$ https: / / www . upwork. com
} 
scribed in this section, i.e. the Upwork teachers rated human-written stories higher for coherence, relevance, and likability than the GPT- 2 generated stories (all $p$ 's $<0.001$ ). Interestingly, their IAA was higher than the English teachers recruited from the authors' personal networks. The details of this experiment are provided in the Appendix B.

\section{Related Work}

Our work is related to previous studies of human evaluation of text quality as well as collecting judgments using Amazon Mechanical Turk.

Human evaluation of text quality: Most previous studies on human evaluation concentrate on constrained generation domains, such as machine translation (Guzmán et al., 2015; Graham et al., 2017; Toral et al., 2018; Castilho, 2021) or summarization (Gillick and Liu, 2010; Iskender et al., 2020). Other studies evaluate very short, often one sentence long, outputs (Grundkiewicz et al., 2015; Mori et al., 2019; Khashabi et al., 2021).

Even professional translators struggle when evaluating longer machine translated texts (Castilho, 2021). Creative texts, such as stories, are less constrained than translated texts, but researchers continue to employ crowd workers to evaluate creative texts, often without evaluating reference texts (see Section 2). Previous studies have asked workers to choose from (Mori et al., 2019) or distinguish between human-written and machine-generated texts (Garbacea et al., 2019; Ippolito et al., 2020; Clark et al., 2021).

Data collection using AMT: Many previous works raise concerns about the reliability of data collected on AMT (Necka et al., 2016; Matherly, 2019; Ahler et al., 2020). Reluctance of requesters to reject HITs leads to positive bias in workers' qualifications (Matherly, 2019). Furthermore, a large number of responses are provided by small number of productive workers (Fort et al., 2011; Robinson et al., 2019). Researchers also report an increasing number of workers use VPNs to mask their location (Bauer et al., 2020) and contribute lower-quality data (Moss and Litman; Ahler et al., 2020). Hence, simple quality control measures, such as approval rate or the country of residence as suggested in (Berinsky et al., 2012), may not be sufficient to effectively filter workers who are spamming a task.

\section{Recommendations \& Conclusion}

Our experiments show that evaluating open-ended generated text is an incredibly challenging task even for expert raters. While AMT is a convenient and affordable solution, we observe that high variance between workers, poor calibration, and cognitively-demanding tasks can lead researchers to draw misleading scientific conclusions (e.g., that human-written text is "worse" than GPT-2's). Simple fixes such as adding strict worker qualifications do not address the root of the problem. As such, we recommend future AMT evaluations implement additional quality control mechanisms (some of which require custom task setups on external servers) such as (1) filtering workers by observed time spent per HIT rather than WorkTimeInSeconds, (2) specifying a maximum number of items per worker, (3) employing a pre-task language proficiency test, and (4) providing training HITs to allow workers to calibrate their ratings. Furthermore, we show that researchers can improve rating calibration by presenting machine-generated text alongside human reference text. That said, expert raters such as linguists or language teachers should be used whenever possible as they have already been trained to evaluate written text, and it is not much more expensive (it cost us $\$ 144$ to rate 200 stories with AMT vs. $\$ 187.50$ with English teachers vs. \$262.5 with Upwork.

\section{Ethical Considerations}

As with all research that makes use of human subjects, we must carefully reflect on our methodology to minimize the risk of harm to those we ask to evaluate open-ended texts. Specifically, texts from social media sites like Reddit may contain racist, sexist, and other forms of vulgar content. Additionally, neural language models like GPT2 , which have been trained on open domain text crawled from the web, have been shown to generate similarly offensive content. As such, we advocate adequately warning any humans who take part in open-ended text evaluation of the potential for such harms (as we did in our research).

Additionally, crowd workers are frequently underpaid for their labor, which harms both the quality of the research, and more importantly, the ability of these crowd workers to earn an adequate living. As such, we report our hourly wage for both crowd workers and experts. We ensure that crowd workers earn at least $\$ 14$ per hour by assuming $50-55$ 
seconds per HIT (though on average our crowd workers were paid substantially higher due to the low average time to completion on each HIT). Our experts averaged around $\$ 20$ per hour (not counting mediation).

\section{Acknowledgments}

We thank the reviewers for their insightful comments. We would also like to thank the UMass NLP group for the great advice on the draft of this paper. We are grateful to the English teachers recruited from Upwork and from the authors personal network, as well as workers on AMT, for their help in the story evaluation. MK is supported by the Chan Zuckerberg Initiative under the project Scientific Knowledge Base Construction. NA and MI are supported by award IIS-1955567 from the National Science Foundation (NSF).

\section{References}

Douglas J. Ahler, Carolyn E. Roush, and Gaurav Sood 2020. Replication data for: "the micro-task market for lemons: Data quality on Amazon's Mechanical Turk".

Nader Akoury, Shufan Wang, Josh Whiting, Stephen Hood, Nanyun Peng, and Mohit Iyyer. 2020. STORIUM: A Dataset and Evaluation Platform for Machine-in-the-Loop Story Generation. In Proceedings of the 2020 Conference on Empirical Methods in Natural Language Processing (EMNLP), pages 6470-6484, Online. Association for Computational Linguistics.

Milad Alshomary, Shahbaz Syed, Martin Potthast, and Henning Wachsmuth. 2020. Target inference in argument conclusion generation. In Proceedings of the 58th Annual Meeting of the Association for Computational Linguistics, pages 4334-4345, Online. Association for Computational Linguistics.

Brian Bauer, Kristy L. Larsen, Nicole Caulfield, Domynic Elder, Sara Jordan, and Daniel Capron. 2020. Review of best practice recommendations for ensuring high quality data with amazon's mechanical turk.

Adam J. Berinsky, Gregory A. Huber, and Gabriel S Lenz. 2012. Evaluating online labor markets for experimental research: Amazon.com's mechanical turk. Political Analysis, 20(3):351-368.

Antoine Bosselut, Asli Celikyilmaz, Xiaodong $\mathrm{He}$ Jianfeng Gao, Po-Sen Huang, and Yejin Choi. 2018 Discourse-aware neural rewards for coherent text generation. In Proceedings of the 2018 Conference of the North American Chapter of the Association for Computational Linguistics: Human Language
Technologies, Volume 1 (Long Papers), pages 173184, New Orleans, Louisiana. Association for Computational Linguistics.

Faeze Brahman and Snigdha Chaturvedi. 2020. Modeling protagonist emotions for emotion-aware storytelling. In Proceedings of the 2020 Conference on Empirical Methods in Natural Language Processing (EMNLP), pages 5277-5294, Online. Association for Computational Linguistics.

D. Card, Peter Henderson, Urvashi Khandelwal, Robin Jia, Kyle Mahowald, and Dan Jurafsky. 2020. With little power comes great responsibility. In EMNLP.

Sheila Castilho. 2021. Towards document-level human MT evaluation: On the issues of annotator agreement, effort and misevaluation. In Proceedings of the Workshop on Human Evaluation of NLP Systems (HumEval), pages 34-45, Online. Association for Computational Linguistics.

Tuhin Chakrabarty, Debanjan Ghosh, Smaranda Muresan, and Nanyun Peng. 2020a. R^3: Reverse, retrieve, and rank for sarcasm generation with commonsense knowledge. In Proceedings of the 58th Annual Meeting of the Association for Computational Linguistics, pages 7976-7986, Online. Association for Computational Linguistics.

Tuhin Chakrabarty, Smaranda Muresan, and Nanyun Peng. 2020b. Generating similes effortlessly like a pro: A style transfer approach for simile generation. In Proceedings of the 2020 Conference on Empirical Methods in Natural Language Processing (EMNLP), pages 6455-6469, Online. Association for Computational Linguistics.

Elizabeth Clark, Tal August, Sofia Serrano, Nikita Haduong, Suchin Gururangan, and Noah A. Smith. 2021. All that's 'human' is not gold: Evaluating human evaluation of generated text. In Proceedings of the 59th Annual Meeting of the Association for Computational Linguistics and the 11th International Joint Conference on Natural Language Processing (Volume 1: Long Papers), pages 7282-7296, Online. Association for Computational Linguistics.

Elizabeth Clark, Yangfeng Ji, and Noah A. Smith. 2018. Neural text generation in stories using entity representations as context. In Proceedings of the 2018 Conference of the North American Chapter of the Association for Computational Linguistics: Human Language Technologies, Volume 1 (Long Papers), pages 2250-2260, New Orleans, Louisiana. Association for Computational Linguistics.

Jacob Cohen. 1988. Statistical power analysis for the behavioral sciences. Routledge.

Chris Donahue, Mina Lee, and Percy Liang. 2020. Enabling language models to fill in the blanks. In Proceedings of the 58th Annual Meeting of the Association for Computational Linguistics, pages 24922501, Online. Association for Computational Linguistics. 
Angela Fan, Mike Lewis, and Yann Dauphin. 2018. Hierarchical neural story generation. In Association for Computational Linguistics.

Angela Fan, Mike Lewis, and Yann Dauphin. 2019 Strategies for structuring story generation. In Proceedings of the 57th Annual Meeting of the Association for Computational Linguistics, pages 2650 2660, Florence, Italy. Association for Computational Linguistics.

Zhiyuan Fang, Tejas Gokhale, Pratyay Banerjee, Chitta Baral, and Yezhou Yang. 2020. Video2Commonsense: Generating commonsense descriptions to enrich video captioning. In Proceedings of the 2020 Conference on Empirical Methods in Natural Language Processing (EMNLP), pages 840-860, Online. Association for Computational Linguistics.

Karën Fort, Gilles Adda, and K. Bretonnel Cohen 2011. Amazon mechanical turk: Gold mine or coal mine? Computational Linguistics, 37(2):413-420.

Cristina Garbacea, Samuel Carton, Shiyan Yan, and Qiaozhu Mei. 2019. Judge the judges: A largescale evaluation study of neural language models for online review generation. In Proceedings of the 2019 Conference on Empirical Methods in Natural Language Processing and the 9th International Joint Conference on Natural Language Processing (EMNLP-IJCNLP), pages 3968-3981, Hong Kong, China. Association for Computational Linguistics.

Dan Gillick and Yang Liu. 2010. Non-expert evaluation of summarization systems is risky. In Proceedings of the NAACL HLT 2010 Workshop on Creating Speech and Language Data with Amazon's Mechanical Turk, pages 148-151, Los Angeles. Association for Computational Linguistics.

Seraphina Goldfarb-Tarrant, Tuhin Chakrabarty, Ralph Weischedel, and Nanyun Peng. 2020. Content planning for neural story generation with aristotelian rescoring. In Proceedings of the 2020 Conference on Empirical Methods in Natural Language Processing (EMNLP), pages 4319-4338, Online. Association for Computational Linguistics.

Philip John Gorinski and Mirella Lapata. 2018. What's this movie about? a joint neural network architecture for movie content analysis. In Proceedings of the 2018 Conference of the North American Chapter of the Association for Computational Linguistics: Human Language Technologies, Volume 1 (Long Papers), pages 1770-1781, New Orleans, Louisiana. Association for Computational Linguistics.

Tanya Goyal and Greg Durrett. 2020. Neural syntactic preordering for controlled paraphrase generation. In Proceedings of the 58th Annual Meeting of the Association for Computational Linguistics, pages 238252, Online. Association for Computational Linguistics.
Yvette Graham, Timothy Baldwin, Alistair Moffat, and Justin Zobel. 2017. Can machine translation systems be evaluated by the crowd alone. Natural Language Engineering, 23(1):3-30.

Roman Grundkiewicz, Marcin Junczys-Dowmunt, and Edward Gillian. 2015. Human evaluation of grammatical error correction systems. In EMNLP.

Jian Guan, Fei Huang, Zhihao Zhao, Xiaoyan Zhu, and Minlie Huang. 2020. A knowledge-enhanced pretraining model for commonsense story generation. Transactions of the Association for Computational Linguistics, 8:93-108.

Francisco Guzmán, Ahmed Abdelali, Irina Temnikova, Hassan Sajjad, and Stephan Vogel. 2015. How do humans evaluate machine translation. In Proceedings of the Tenth Workshop on Statistical Machine Translation. Association for Computational Linguistics.

He He, Nanyun Peng, and Percy Liang. 2019. Pun generation with surprise. In Proceedings of the 2019 Conference of the North American Chapter of the Association for Computational Linguistics: Human Language Technologies, Volume 1 (Long and Short Papers), pages 1734-1744, Minneapolis, Minnesota. Association for Computational Linguistics.

Allison Hegel, Sudha Rao, Asli Celikyilmaz, and Bill Dolan. 2020. Substance over Style: DocumentLevel Targeted Content Transfer. In Proceedings of the 2020 Conference on Empirical Methods in Natural Language Processing (EMNLP), pages 64856504, Online. Association for Computational Linguistics.

Ari Holtzman, Jan Buys, Li Du, Maxwell Forbes, and Yejin Choi. 2019. The curious case of neural text degeneration. In International Conference on Learning Representations.

Ari Holtzman, Jan Buys, Maxwell Forbes, Antoine Bosselut, David Golub, and Yejin Choi. 2018. Learning to write with cooperative discriminators. In Proceedings of the 56th Annual Meeting of the Association for Computational Linguistics (Volume 1: Long Papers), pages 1638-1649, Melbourne, Australia. Association for Computational Linguistics.

David M. Howcroft, Anya Belz, Miruna-Adriana Clinciu, Dimitra Gkatzia, Sadid A. Hasan, Saad Mahamood, Simon Mille, Emiel van Miltenburg, Sashank Santhanam, and Verena Rieser. 2020 Twenty years of confusion in human evaluation: NLG needs evaluation sheets and standardised definitions. In Proceedings of the 13th International Conference on Natural Language Generation, pages 169-182, Dublin, Ireland. Association for Computational Linguistics.

Ting-Yao Hsu, Chieh-Yang Huang, Yen-Chia Hsu, and Ting-Hao Huang. 2019. Visual story post-editing. In Proceedings of the 57th Annual Meeting of the 
Association for Computational Linguistics, pages 6581-6586, Florence, Italy. Association for Computational Linguistics.

Daphne Ippolito, Daniel Duckworth, Chris CallisonBurch, and Douglas Eck. 2020. Automatic detection of generated text is easiest when humans are fooled. In Proceedings of the 58th Annual Meeting of the Association for Computational Linguistics, pages 1808-1822, Online. Association for Computational Linguistics.

Daphne Ippolito, David Grangier, Chris CallisonBurch, and Douglas Eck. 2019. Unsupervised hierarchical story infilling. In Proceedings of the First Workshop on Narrative Understanding, pages 3743, Minneapolis, Minnesota. Association for Computational Linguistics.

Neslihan Iskender, Tim Polzehl, and Sebastian Möller 2020. Best practices for crowd-based evaluation of German summarization: Comparing crowd, expert and automatic evaluation. In Proceedings of the First Workshop on Evaluation and Comparison of NLP Systems, pages 164-175, Online. Association for Computational Linguistics.

Chao Jiang, Mounica Maddela, Wuwei Lan, Yang Zhong, and Wei Xu. 2020. Neural CRF model for sentence alignment in text simplification. In Proceedings of the 58th Annual Meeting of the Association for Computational Linguistics, pages 79437960, Online. Association for Computational Linguistics.

Ryan Kennedy, Scott Clifford, Tyler Burleigh, Philip D. Waggoner, Ryan Jewell, and Nicholas J. G. Winter. 2020. The shape of and solutions to the mturk quality crisis. Political Science Research and Methods, 8(4):614-629.

Daniel Khashabi, Gabriel Stanovsky, Jonathan Bragg, Nicholas Lourie, Jungo Kasai, Yejin Choi, Noah A. Smith, and Daniel S. Weld. 2021. Genie: A leaderboard for human-in-the-loop evaluation of text generation.

Kalpesh Krishna, John Wieting, and Mohit Iyyer. 2020. Reformulating unsupervised style transfer as paraphrase generation. In Proceedings of the 2020 Conference on Empirical Methods in Natural Language Processing (EMNLP), pages 737-762, Online. Association for Computational Linguistics.

Reno Kriz, João Sedoc, Marianna Apidianaki, Carolina Zheng, Gaurav Kumar, Eleni Miltsakaki, and Chris Callison-Burch. 2019. Complexity-weighted loss and diverse reranking for sentence simplification. In Proceedings of the 2019 Conference of the North American Chapter of the Association for Computational Linguistics: Human Language Technologies, Volume 1 (Long and Short Papers), pages 3137-3147, Minneapolis, Minnesota. Association for Computational Linguistics.
Juncen Li, Robin Jia, He He, and Percy Liang. 2018. Delete, retrieve, generate: a simple approach to sentiment and style transfer. In Proceedings of the 2018 Conference of the North American Chapter of the Association for Computational Linguistics: Human Language Technologies, Volume 1 (Long Papers), pages 1865-1874, New Orleans, Louisiana. Association for Computational Linguistics.

Chin-Yew Lin. 2004. Rouge: A package for automatic evaluation of summaries. In $A C L$.

Kevin Lin, Ming-Yu Liu, Ming-Ting Sun, and Jan Kautz. 2020. Learning to generate multiple style transfer outputs for an input sentence. In Proceedings of the Fourth Workshop on Neural Generation and Translation, pages 10-23, Online. Association for Computational Linguistics.

Hui Liu, Qingyu Yin, and William Yang Wang. 2019. Towards explainable NLP: A generative explanation framework for text classification. In Proceedings of the 57th Annual Meeting of the Association for Computational Linguistics, pages 5570-5581, Florence, Italy. Association for Computational Linguistics.

Jonathan Mallinson, Rico Sennrich, and Mirella Lapata. 2020. Zero-shot crosslingual sentence simplification. In Proceedings of the 2020 Conference on Empirical Methods in Natural Language Processing (EMNLP), pages 5109-5126, Online. Association for Computational Linguistics.

Huanru Henry Mao, Bodhisattwa Prasad Majumder, Julian McAuley, and Garrison Cottrell. 2019. Improving neural story generation by targeted common sense grounding. In Proceedings of the 2019 Conference on Empirical Methods in Natural Language Processing and the 9th International Joint Conference on Natural Language Processing (EMNLPIJCNLP), pages 5988-5993, Hong Kong, China. Association for Computational Linguistics.

Pedro Henrique Martins, Zita Marinho, and André F. T. Martins. 2020. Sparse text generation. In Proceedings of the 2020 Conference on Empirical Methods in Natural Language Processing (EMNLP), pages 4252-4273, Online. Association for Computational Linguistics.

Ted Matherly. 2019. A panel for lemons? positivity bias, reputation systems and data quality on MTurk. European Journal of Marketing, 53(2):195-223.

Remi Mir, Bjarke Felbo, Nick Obradovich, and Iyad Rahwan. 2019. Evaluating style transfer for text. In Proceedings of the 2019 Conference of the North American Chapter of the Association for Computational Linguistics: Human Language Technologies, Volume 1 (Long and Short Papers), pages 495-504, Minneapolis, Minnesota. Association for Computational Linguistics.

Yatri Modi and Natalie Parde. 2019. The steep road to happily ever after: an analysis of current visual 
storytelling models. In Proceedings of the Second Workshop on Shortcomings in Vision and Language, pages 47-57, Minneapolis, Minnesota. Association for Computational Linguistics.

Yusuke Mori, Hiroaki Yamane, Yusuke Mukuta, and Tatsuya Harada. 2019. Toward a better story end: Collecting human evaluation with reasons. In Proceedings of the 12th International Conference on Natural Language Generation, pages 383-390, Tokyo, Japan. Association for Computational Linguistics.

Aaron Moss and Leib Litman. After the bot scare: Understanding what's been happening with data collection on mturk and how to stop it. Accessed on 15.04.2021.

Elizabeth A. Necka, Stephanie Cacioppo, Greg J. Norman, and John T. Cacioppo. 2016. Measuring the prevalence of problematic respondent behaviors among MTurk, campus, and community participants. PLOS ONE, 11(6):e0157732.

Kishore Papineni, Salim Roukos, Todd Ward, and WeiJing Zhu. 2002. Bleu: a method for automatic evaluation of machine translation. In Proceedings of the 40th annual meeting on association for computational linguistics, pages 311-318. Association for Computational Linguistics.

Baolin Peng, Xiujun Li, Lihong Li, Jianfeng Gao, Asli Celikyilmaz, Sungjin Lee, and Kam-Fai Wong. 2017. Composite task-completion dialogue policy learning via hierarchical deep reinforcement learning. In Proceedings of the 2017 Conference on Empirical Methods in Natural Language Processing. Association for Computational Linguistics.

Nanyun Peng, Marjan Ghazvininejad, Jonathan May, and Kevin Knight. 2018a. Towards controllable story generation. In Proceedings of the First Workshop on Storytelling. Association for Computational Linguistics.

Nanyun Peng, Marjan Ghazvininejad, Jonathan May, and Kevin Knight. 2018b. Towards controllable story generation. In Proceedings of the First Workshop on Storytelling, pages 43-49, New Orleans, Louisiana. Association for Computational Linguistics.

Pouya Pezeshkpour, Liyan Chen, and Sameer Singh. 2018. Embedding multimodal relational data for knowledge base completion. In Proceedings of the 2018 Conference on Empirical Methods in Natural Language Processing, pages 3208-3218, Brussels, Belgium. Association for Computational Linguistics.

Lianhui Qin, Antoine Bosselut, Ari Holtzman, Chandra Bhagavatula, Elizabeth Clark, and Yejin Choi. 2019. Counterfactual story reasoning and generation. In Proceedings of the 2019 Conference on Empirical Methods in Natural Language Processing and the 9th International Joint Conference on Natural Language Processing (EMNLP-IJCNLP), pages 50435053, Hong Kong, China. Association for Computational Linguistics.

Lianhui Qin, Vered Shwartz, Peter West, Chandra Bhagavatula, Jena D. Hwang, Ronan Le Bras, Antoine Bosselut, and Yejin Choi. 2020. Back to the future: Unsupervised backprop-based decoding for counterfactual and abductive commonsense reasoning. In Proceedings of the 2020 Conference on Empirical Methods in Natural Language Processing (EMNLP), pages 794-805, Online. Association for Computational Linguistics.

Alec Radford, Jeffrey Wu, Rewon Child, David Luan, Dario Amodei, and Ilya Sutskever. 2019. Language models are unsupervised multitask learners.

Sudha Rao and Joel Tetreault. 2018. Dear sir or madam, may I introduce the GYAFC dataset: Corpus, benchmarks and metrics for formality style transfer. In Proceedings of the 2018 Conference of the North American Chapter of the Association for Computational Linguistics: Human Language Technologies, Volume 1 (Long Papers), pages 129-140, New Orleans, Louisiana. Association for Computational Linguistics.

Hannah Rashkin, Asli Celikyilmaz, Yejin Choi, and Jianfeng Gao. 2020. PlotMachines: Outlineconditioned generation with dynamic plot state tracking. In Proceedings of the 2020 Conference on Empirical Methods in Natural Language Processing (EMNLP), pages 4274-4295, Online. Association for Computational Linguistics.

Ehud Reiter. 2018. A structured review of the validity of BLEU. Computational Linguistics, 44(3):393401.

Jonathan Robinson, Cheskie Rosenzweig, Aaron J. Moss, and Leib Litman. 2019. Tapped out or barely tapped? recommendations for how to harness the vast and largely unused potential of the mechanical turk participant pool. PLOS ONE, 14(12):e0226394.

Abigail See, Aneesh Pappu, Rohun Saxena, Akhila Yerukola, and Christopher D Manning. 2019. Do massively pretrained language models make better storytellers? In Conference on Computational Natural Language Learning.

Dinghan Shen, Asli Celikyilmaz, Yizhe Zhang, Liqun Chen, Xin Wang, Jianfeng Gao, and Lawrence Carin. 2019. Towards generating long and coherent text with multi-level latent variable models. In Proceedings of the 57th Annual Meeting of the Association for Computational Linguistics, pages 2079-2089, Florence, Italy. Association for Computational Linguistics.

Akhilesh Sudhakar, Bhargav Upadhyay, and Arjun Maheswaran. 2019. "transforming" delete, retrieve, 
generate approach for controlled text style transfer. In Proceedings of the 2019 Conference on Empirical Methods in Natural Language Processing and the 9th International Joint Conference on Natural Language Processing (EMNLP-IJCNLP), pages 32693279 , Hong Kong, China. Association for Computational Linguistics.

Antonio Toral, Sheila Castilho, $\mathrm{Ke} \mathrm{Hu}$, and Andy Way. 2018. Attaining the unattainable? reassessing claims of human parity in neural machine translation.

Lifu Tu, Xiaoan Ding, Dong Yu, and Kevin Gimpel. 2019. Generating diverse story continuations with controllable semantics. In Proceedings of the $3 \mathrm{rd}$ Workshop on Neural Generation and Translation, pages 44-58, Hong Kong. Association for Computational Linguistics.

Chris van der Lee, Albert Gatt, Emiel van Miltenburg, and Emiel Krahmer. 2021. Human evaluation of automatically generated text: Current trends and best practice guidelines. Computer Speech \& Language, 67:101151

K. Wessling, J. Huber, and O. Netzer. 2017. Mturk character misrepresentation: Assessment and solutions. Journal of Consumer Research, 44:211-230.

Thomas Wolf, Lysandre Debut, Victor Sanh, Julien Chaumond, Clement Delangue, Anthony Moi, Pierric Cistac, Tim Rault, Remi Louf, Morgan Funtowicz, Joe Davison, Sam Shleifer, Patrick von Platen, Clara Ma, Yacine Jernite, Julien Plu, Canwen Xu, Teven Le Scao, Sylvain Gugger, Mariama Drame, Quentin Lhoest, and Alexander Rush. 2020. Transformers: State-of-the-art natural language processing. In Proceedings of the 2020 Conference on Empirical Methods in Natural Language Processing: System Demonstrations, pages 38-45, Online. Association for Computational Linguistics.

Peng Xu, Mostofa Patwary, Mohammad Shoeybi, Raul Puri, Pascale Fung, Anima Anandkumar, and Bryan Catanzaro. 2020. MEGATRON-CNTRL: Controllable story generation with external knowledge using large-scale language models. In Proceedings of the 2020 Conference on Empirical Methods in Natural Language Processing (EMNLP), pages 28312845, Online. Association for Computational Linguistics.

Wei Yang, Yuqing Xie, Aileen Lin, Xingyu Li, Luchen Tan, Kun Xiong, Ming Li, and Jimmy Lin. 2019. End-to-end open-domain question answering with BERTserini. In Proceedings of the 2019 Conference of the North American Chapter of the Association for Computational Linguistics (Demonstrations), pages 72-77, Minneapolis, Minnesota. Association for Computational Linguistics.

Zhiwei Yu, Jiwei Tan, and Xiaojun Wan. 2018. A neural approach to pun generation. In Proceedings of the 56th Annual Meeting of the Association for
Computational Linguistics (Volume 1: Long Papers), pages 1650-1660, Melbourne, Australia. Association for Computational Linguistics.

Zhiwei Yu and Xiaojun Wan. 2019. How to avoid sentences spelling boring? towards a neural approach to unsupervised metaphor generation. In Proceedings of the 2019 Conference of the North American Chapter of the Association for Computational Linguistics: Human Language Technologies, Volume 1 (Long and Short Papers), pages 861-871, Minneapolis, Minnesota. Association for Computational Linguistics.

Zhiwei Yu, Hongyu Zang, and Xiaojun Wan. 2020. Routing enforced generative model for recipe generation. In Proceedings of the 2020 Conference on Empirical Methods in Natural Language Processing (EMNLP), pages 3797-3806, Online. Association for Computational Linguistics.

Hongyu Zang, Zhiwei Yu, and Xiaojun Wan. 2019. Automated chess commentator powered by neural chess engine. In Proceedings of the 57th Annual Meeting of the Association for Computational Linguistics, pages 5952-5961, Florence, Italy. Association for Computational Linguistics.

Rui Zhang and Joel Tetreault. 2019. This email could save your life: Introducing the task of email subject line generation. In Proceedings of the 57th Annual Meeting of the Association for Computational Linguistics, pages 446-456, Florence, Italy. Association for Computational Linguistics.

A. Çelikyilmaz, Elizabeth Clark, and Jianfeng Gao. 2020. Evaluation of text generation: A survey. ArXiv, abs/2006.14799. 


\section{Qualifications}

\begin{tabular}{|c|c|c|c|c|}
\hline Approval Rate & $90 \%(4)$ & $95 \%(3)$ & $97 \%(1)$ & $99 \%(1)$ \\
\hline Accepted HITs & $500(1)$ & $1000(5)$ & $5000(1)$ & \\
\hline English & Resident (6) & Native Speaker (4) & Self-reported (1) & \\
\hline Other & \multicolumn{2}{|c|}{ Complete all ratings $(I)$} & \multicolumn{2}{|c|}{ Passed quality check ( $(1)$} \\
\hline Payment & $\$ 0.05-\$ 2$ per $\mathrm{H}$ & $\mathrm{T}(8)$ & $\$ 12-\$ 20$ per hou & $(2)$ \\
\hline Number of Items & $<100$ items $(9)$ & 100 items $(14)$ & $>100$ items $(20)$ & not reported (5) \\
\hline Number of Raters & $<3$ raters $(3)$ & 3 raters $(16)$ & 5 raters $(13)$ & $>5$ raters $(3)$ \\
\hline Likert Scale & 3-point (4) & 4-point (3) & 5-point (24) & 6-point (1) \\
\hline Ranking Task & two texts (19) & more texts (4) & & \\
\hline
\end{tabular}

Table A1: Results of the survey from Section 2. Numbers in brackets refer to the number of papers/experiments which employed the given measure.

\section{B Collecting Ratings on Upwork}

\section{A Questions Used for the Survey}

The paper survey described in Section 2 included the following questions:

- type of the task

- length of rated text

- rated attributes

- rating scale

- labels used for ratings

- definitions of each attribute

- instructions provided to raters

- qualifications and quality control measures employed

- number of rated items

- number of rated systems

We also hired three teachers using the freelancing platform Upwork ${ }^{1}$. The teachers were paid $\$ 175$ to evaluate the same 200 human-written stories and 200 GPT-2 generated stories. They were asked to perform the ratings on the AMT platform in order to use the same interface as workers on AMT. Similarly to the teachers recruited from the authors' personal network, the teachers recruited on Upwork were asked to rate the 200 human-written stories first and then, after a few days break, provide the ratings for the GPT-2 generated stories. Furthermore, Upwork teachers also held TEFL, ${ }^{2}$ TESOL, ${ }^{3}$ or CELTA ${ }^{13}$ certificates. Table A3 shows mean ratings and agreement for the data collected on Upwork. Similarly to the results described in Section 4 and summarized in Table A5, the average scores for coherence, relevance, and likability are higher for the human-written stories than for the GPT-2 generated stories (see Table A4).

- number of raters per item

- inclusion of ground truth

- monetary compensation

\footnotetext{
${ }^{1}$ https: // www . upwork. com/

${ }^{2}$ Teaching English as a Foreign Language.

${ }^{3}$ Teaching English to Speakers of Other Languages
} 


\begin{tabular}{|c|c|c|c|}
\hline Authors & Year & Title & Venue \\
\hline Akoury et al. & 2020 & STORIUM: A Dataset and Evaluation Platform for Machine-in-the-Loop Story Generation & EMNLP \\
\hline Alshomary et al. & 2020 & Target Inference in Argument Conclusion Generation & ACL \\
\hline Bosselut et al. & 2018 & Discourse-Aware Neural Rewards for Coherent Text Generation & EMNLP \\
\hline Brahman and Chaturvedi & 2020 & Modeling Protagonist Emotions for Emotion-Aware Storytelling & EMNLP \\
\hline Chakrabarty et al. & $2020 \mathrm{~b}$ & Generating similes effortlessly like a Pro: A Style Transfer Approach for Simile Generation & EMNLP \\
\hline Chakrabarty et al. & $2020 \mathrm{a}$ & R3: Reverse, Retrieve, and Rank for Sarcasm Generation with Commonsense Knowledge & ACL \\
\hline Clark et al. & 2018 & Neural Text Generation in Stories Using Entity Representations as Context & NAACL \\
\hline Donahue et al. & 2020 & Enabling Language Models to Fill in the Blanks & ACL \\
\hline Fan et al. & 2018 & Hierarchical Neural Story Generation & $\mathrm{ACL}$ \\
\hline Fang et al. & 2020 & Video2Commonsense: Generating Commonsense Descriptions to Enrich Video Captioning & EMNLP \\
\hline Goldfarb-Tarrant et al. & 2020 & Content Planning for Neural Story Generation with Aristotelian Rescoring & EMNLP \\
\hline Gorinski and Lapata & 2018 & What's this Movie about? A Joint Neural Network Architecture for Movie Content Analysis & NAACL \\
\hline Goyal and Durrett & 2020 & Neural Syntactic Preordering for Controlled Paraphrase Generation & ACL \\
\hline He et al. & 2019 & Pun Generation with Surprise & NAACL \\
\hline Hegel et al. & 2020 & Substance over Style: Document-Level Targeted Content Transfer & EMNLP \\
\hline Holtzman et al. & 2018 & Learning to Write with Cooperative Discriminators & ACL \\
\hline Hsu et al. & 2019 & Visual Story Post-Editing & ACL \\
\hline Ippolito et al. & 2019 & Unsupervised Hierarchical Story Infilling & NAACL \\
\hline Jiang et al. & 2020 & Neural CRF Model for Sentence Alignment in Text Simplification & ACL \\
\hline Krishna et al. & 2020 & Reformulating Unsupervised Style Transfer as Paraphrase Generation & EMNLP \\
\hline Kriz et al. & 2019 & Complexity-Weighted Loss and Diverse Reranking for Sentence Simplification & NAACL \\
\hline Li et al. & 2018 & Delete, Retrieve, Generate: A Simple Approach to Sentiment and Style Transfer & NAACL \\
\hline Lin et al. & 2020 & Learning to Generate Multiple Style Transfer Outputs for an Input Sentence & ACL \\
\hline Liu et al. & 2019 & Towards Explainable NLP: A Generative Explanation Framework for Text Classification & ACL \\
\hline Mallinson et al. & 2020 & Zero-Shot Crosslingual Sentence Simplification & EMNLP \\
\hline Martins et al. & 2020 & Sparse Text Generation & EMNLP \\
\hline Mir et al. & 2019 & Evaluating Style Transfer for Text & NAACL \\
\hline Peng et al. & 2018b & Towards Controllable Story Generation & NAACL \\
\hline Pezeshkpour et al. & 2018 & Embedding Multimodal Relational Data for Knowledge Base Completion & EMNLP \\
\hline Qin et al. & 2020 & Embedding Multimodal Relational Data for Knowledge Base Completion & EMNLP \\
\hline Qin et al. & 2019 & Counterfactual Story Reasoning and Generation & EMNLP \\
\hline Rao and Tetreault & 2018 & Dear Sir or Madam, May I Introduce the GYAFC Dataset: Corpus, Benchmarks and Metrics for Formality Style Transfer & NAACL \\
\hline Rashkin et al. & 2020 & PLOT MACHINES: Outline-Conditioned Generation with Dynamic Plot State Tracking & EMNLP \\
\hline Shen et al. & 2019 & Towards Generating Long and Coherent Text with Multi-Level Latent Variable Models & ACL \\
\hline Sudhakar et al. & 2019 & "Transforming" Delete, Retrieve, Generate Approach for Controlled Text Style Transfer & EMNLP \\
\hline Tu et al. & 2019 & Generating Diverse Story Continuations with Controllable Semantics & EMNLP \\
\hline Tu et al. & 2019 & Can Humor Prediction Datasets be used for Humor Generation? Humorous Headline Generation via Style Transfer & ACL \\
\hline Xu et al. & 2020 & MEGATRON - CNTRL : Controllable Story Generation with External Knowledge Using Large-Scale Language Models & EMNLP \\
\hline Yang et al. & 2019 & An End-to-End Generative Architecture for Paraphrase Generation & EMNLP \\
\hline Modi and Parde & 2019 & The Steep Road to Happily Ever After: An Analysis of Current Visual Storytelling Models & NAACL \\
\hline Yu and Wan & 2019 & How to Avoid Sentences Spelling Boring? Towards a Neural Approach to Unsupervised Metaphor Generation & NAACL \\
\hline Yu et al. & 2018 & A Neural Approach to Pun Generation & ACL \\
\hline Yu et al. & 2020 & Routing Enforced Generative Model for Recipe Generation & EMNLP \\
\hline Zhang and Tetreault & 2019 & This Email Could Save Your Life: Introducing the Task of Email Subject Line Generation & ACL \\
\hline Zang et al. & 2019 & Automated Chess Commentator Powered by Neural Chess Engine & ACL \\
\hline
\end{tabular}

Table A2: List of Surveyed Papers. 


\begin{tabular}{lllllllll}
\hline \multirow{2}{*}{ Experiment description } & \multicolumn{2}{c}{ Grammar } & \multicolumn{2}{c}{ Coherence } & \multicolumn{2}{c}{ Relevance } & \multicolumn{2}{c}{ Likability } \\
& Mean $_{S T D}$ & IAA\% & Mean IAD $_{S T}$ & $\mathrm{IAA}_{\%}$ & Mean $_{S T D}$ & $\mathrm{IAA}_{\%}$ & Mean $_{S T D}$ & $\mathrm{IAA}_{\%}$ \\
\hline Reference & $4.28_{0.78}$ & $0.29_{26}$ & $4.55_{0.66}$ & $0.11_{30.5}$ & $4.25_{0.88}$ & $0.49_{26.4}$ & $4.02_{1.16}$ & $0.01_{14.5}$ \\
GPT-2 & $4.25_{0.82}$ & $0.12_{19}$ & $3.99_{0.98}$ & $0.06_{12.5}$ & $3.02_{1.53}$ & $0.25_{5}$ & $3.68_{1.15}$ & $0.05_{10.5}$ \\
\hline
\end{tabular}

Table A3: Ratings of human-written reference stories and GPT-2 generated stories collected on Upwork. Interannotator agreement (IAA) between the three raters is measured with Krippendorff's $\alpha$ well as the percentage of stories for which all three raters exactly agreed on a rating (the latter is subscripted)

\begin{tabular}{lllllllll}
\hline & mean (human) & mean (GPT-2) & difference & $95 \%$ CI lower & $95 \%$ CI upper & t & df & $p$-val \\
\hline grammar & 4.28 & 4.25 & 0.03 & -0.06 & 0.12 & 0.72 & 1194.9 & 0.47 \\
coherence & 4.55 & 3.99 & 0.56 & 0.46 & 0.65 & 11.46 & 1044.3 & $<\mathbf{0 . 0 0 1}$ \\
relevance & 4.02 & 3.02 & 1.00 & 0.85 & 1.15 & 12.82 & 1103.8 & $<\mathbf{0 . 0 0 1}$ \\
likability & 4.23 & 3.83 & 0.40 & 0.30 & 0.53 & 7.24 & 1143.4 & $<\mathbf{0 . 0 0 1}$ \\
\hline
\end{tabular}

Table A4: Welch's $t$-test for ratings collected on Upwork (human-written stories vs GPT-2 generated stories). Human-written stories were rated higher on coherence, relevance, and likability than GPT-2 generated stories. These results are similar to the one obtained from English teachers described in Section 4.

\section{Details on Post-rating Interviews}

Two mediation meetings were organized with two of the three teachers (due to availability) over Zoom ${ }^{4}$. The teachers were asked to reevaluate 60 stories on which they showed disagreement (3 attributes $\times 10$ stories $\times 2$ types; likability was excluded due to its subjective nature). Each meeting took approximately $2 \mathrm{~h}$ (including a short break) and was led by one of the authors. The teachers were shown one story at a time and were asked to reevaluate it on the given attribute. In about $20 \%$ of the cases, the teachers agreed with each other, suggesting that the previous disagreement was due to honest lapses of judgment. As for the cases where disagreement occurred, each was asked to provide a justification for their ratings. Often hearing the other party's argument enabled them to see the text from a different perspective and understand the ratings of the other person. This process often resulted in them adjusting their own ratings. Common reasons for disagreement which could be resolved during the mediation meeting included: world knowledge, difference in understanding of the prompt and its relation to the text (e.g., prompt enforcing specific style), difference in the way they treated author's comments which were sometimes present at the beginning of the story, and rationalizing connections between the sentences.

After each batch, consisting of ratings of both human-written stories and GPT-2 generated stories, each of the three teachers took part in a short oneon-one interview ( $\sim 10 \mathrm{~min}$ each). They were asked the following questions:
1. How long did it take you to calibrate your ratings?

2. Explain in more detail how you rated coherence/grammar/likability/relevance? What was the process. Did you have to reread the text?

3. How did you calibrate the ratings for coherencelgrammar/likability/relevance? What constituted a 5? What about a 1 ?

4. How often did you take breaks?

5. Which attribute was the easiest to calibrate? Which was the most difficult?

6. Any other comments or suggestions?

Additionally, after the second batch of GPT-2 stories, the teachers were also asked: (1) which batch was better written, (2) which batch included more computer generated stories and to what extent, (3) whether they had to recalibrate their ratings, and (4) whether they would prefer to see both batches at the same time.

\section{Statistical Analysis}

\footnotetext{
${ }^{4}$ https: // zoom.us/
} 


\begin{tabular}{lllllllll}
\hline & mean (human) & mean (GPT-2) & difference & $95 \%$ CI lower & $95 \%$ CI upper & t & df & $p$-val \\
\hline grammar & 4.50 & 4.55 & 0.05 & -0.14 & 0.03 & -1.27 & 1111.7 & 0.21 \\
coherence & 4.38 & 3.73 & 0.65 & 0.53 & 0.77 & 10.63 & 1119.6 & $<\mathbf{0 . 0 0 1}$ \\
relevance & 3.82 & 2.54 & 1.28 & 1.12 & 1.44 & 15.45 & 1190.7 & $<\mathbf{0 . 0 0 1}$ \\
likability & 3.69 & 2.96 & 0.73 & 0.57 & 0.89 & 9.16 & 1182 & $<\mathbf{0 . 0 0 1}$ \\
\hline
\end{tabular}

Table A5: Welch's $t$-test for ratings collected in the experiment described in Section 4 (teachers' ratings). Humanwritten stories were rated higher for coherence, relevance, and likability than GPT-2 generated stories.

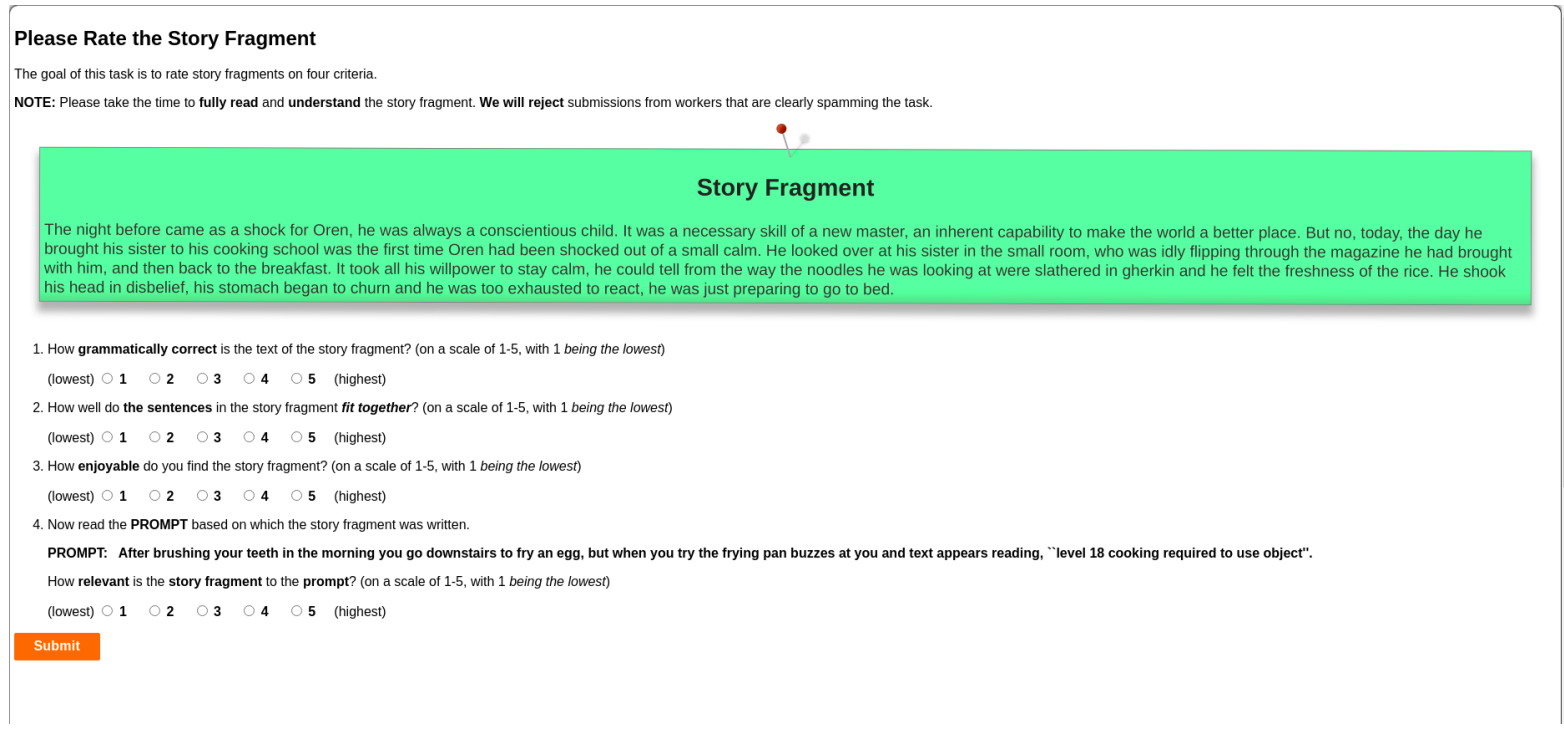

Figure A1: AMT interface for evaluation of one story.

\begin{tabular}{lrrrrrr}
\hline & Df & Sum Sq & Mean Sq & F value & $\operatorname{Pr}(>\mathrm{F})$ & $\eta_{p}^{2}$ \\
\hline Group & 3 & 13.63 & 4.54 & 4.90 & $\mathbf{0 . 0 0 2}$ & 0.01 \\
Residuals & 2396 & 2223.51 & 0.93 & & & \\
\hline
\end{tabular}

Table A6: One-way ANOVA investigating the effect of group (Day 1, Day 2, Day 3, and workers from non-Englishspeaking countries) on the ratings of grammar of the reference texts. Partial eta squared $\left(\eta_{p}^{2}\right)$ is provided for the effect size $\left(\eta_{p}^{2}=0.01\right.$ indicates small effect size; $\eta_{p}^{2}=0.06$ indicates medium effect size; $\eta_{p}^{2}=0.14$ indicates large effect size (Cohen, 1988)).

\begin{tabular}{rrrr}
\hline & Day 1 & Day 2 & Day 3 \\
\hline Day 2 & 0.08 & & \\
Day 3 & 1.00 & 0.20 & \\
NNS & $\mathbf{0 . 0 1}$ & 1.00 & $\mathbf{0 . 0 3}$ \\
\hline
\end{tabular}

Table A7: Pairwise post-hoc test with Bonferroni adjustment for the ratings of grammar between Day 1, Day 2, Day 3, and non-English speaking countries (NNS). The numbers provided in the table are $p$-values for the given pairwise comparison. Grammar ratings provided by the workers from non-English speaking countries are significantly different from ratings provided by the workers from English-speaking countries on Day 1 and Day $3^{5}$

\begin{tabular}{lrrrrrr}
\hline & Df & Sum Sq & Mean Sq & F value & $\operatorname{Pr}(>\mathrm{F}))$ & $\eta_{p}^{2}$ \\
\hline Group & 3 & 161.28 & 53.76 & 51.35 & $<\mathbf{0 . 0 0 1}$ & 0.06 \\
Residuals & 2396 & 2508.21 & 1.05 & & & \\
\hline
\end{tabular}

Table A8: One-way ANOVA investigating the effect of group (Day 1, Day 2, Day 3, and workers from non-Englishspeaking countries) on the ratings of coherence of the reference texts. Partial eta squared $\left(\eta_{p}^{2}\right)$ is provided for the effect size $\left(\eta_{p}^{2}=0.01\right.$ indicates small effect size; $\eta_{p}^{2}=0.06$ indicates medium effect size; $\eta_{p}^{2}=0.14$ indicates large effect size (Cohen, 1988)). 


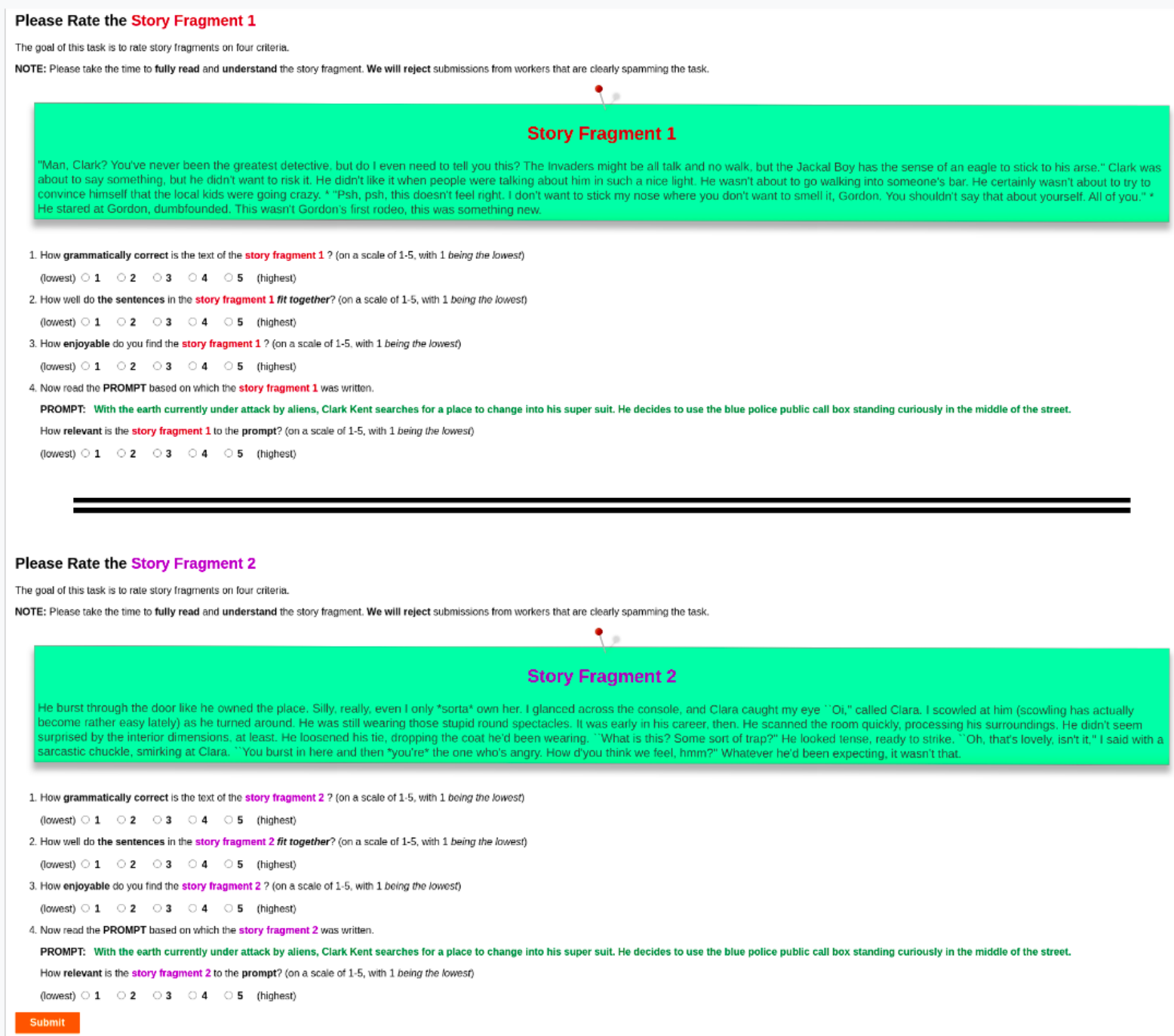

Figure A2: AMT interface for evaluation of both types of stories (GPT-2 and human reference).

\begin{tabular}{rrrr}
\hline & Day 1 & Day 2 & Day 3 \\
\hline Day 2 & 1.00 & & \\
Day 3 & $<\mathbf{0 . 0 0 1}$ & $<\mathbf{0 . 0 0 1}$ & \\
NNS & $<\mathbf{0 . 0 0 1}$ & $<\mathbf{0 . 0 0 1}$ & $\mathbf{0 . 0 2}$ \\
\hline
\end{tabular}

Table A9: Pairwise post hoc test with Bonferroni adjustment for the ratings of coherence between Day 1, Day 2, Day 3, and non-English speaking countries (NNS). The numbers provided in the table are $p$-values for the given pairwise comparison. Ratings of coherence provided by raters from non-English speaking countries are significantly different from ratings of workers from English-speaking countries. Furthermore, there are some difference between Day 1, Day 2, and Day 2.

\begin{tabular}{lrrrrrr}
\hline & Df & Sum Sq & Mean Sq & F value & $\operatorname{Pr}(>\mathrm{F})$ & $\eta_{p}^{2}$ \\
\hline Group & 3 & 89.99 & 30.00 & 19.92 & $<\mathbf{0 . 0 0 1}$ & 0.02 \\
Residuals & 2396 & 3607.85 & 1.51 & & & \\
\hline
\end{tabular}

Table A10: One-way ANOVA investigating the effect of group (Day 1, Day 2, Day 3, and workers from nonEnglish-speaking countries) on the ratings of relevance of the reference texts. Partial eta squared $\left(\eta_{p}^{2}\right)$ is provided for the effect size $\left(\eta_{p}^{2}=0.01\right.$ indicates small effect size; $\eta_{p}^{2}=0.06$ indicates medium effect size; $\eta_{p}^{2}=0.14$ indicates large effect size (Cohen, 1988)). 


\begin{tabular}{rrrr}
\hline & Day 1 & Day 2 & Day 3 \\
\hline Day 2 & $\mathbf{0 . 0 1}$ & & \\
Day 3 & 1.00 & 0.14 & \\
NNS & $<\mathbf{0 . 0 0 1}$ & $<\mathbf{0 . 0 0 1}$ & $<\mathbf{0 . 0 0 1}$ \\
\hline
\end{tabular}

Table A11: Pairwise post hoc test with Bonferroni adjustment for the ratings of relevance between Day 1, Day 2, Day 3, and non-English speaking countries (NNS). The numbers provided in the table are $p$-values for the given pairwise comparison. Ratings obtained from workers from non-English speaking countries differ significantly from ratings obtained from workers from English-speaking countries on Day 1, Day 2, and Day 3. Furthermore, there is a significant difference between ratings collected on Day 1 and Day 2.

\begin{tabular}{lrrrrrr}
\hline & Df & Sum Sq & Mean Sq & F value & $\operatorname{Pr}(>\mathrm{F})$ & $\eta_{p}^{2}$ \\
\hline Group & 3 & 62.70 & 20.90 & 15.89 & $<\mathbf{0 . 0 0 1}$ & 0.02 \\
Residuals & 2396 & 3151.22 & 1.32 & & & \\
\hline
\end{tabular}

Table A12: One-way ANOVA investigating the effect of group (Day 1, Day 2, Day 3, and workers from nonEnglish-speaking countries) on the ratings of likability of the reference texts. Partial eta squared $\left(\eta_{p}^{2}\right)$ is provided for the effect size $\left(\eta_{p}^{2}=0.01\right.$ indicates small effect size; $\eta_{p}^{2}=0.06$ indicates medium effect size; $\eta_{p}^{2}=0.14$ indicates large effect size (Cohen, 1988)).

\begin{tabular}{rrrr}
\hline & Day 1 & Day 2 & Day 3 \\
\hline Day 2 & $<\mathbf{0 . 0 0 1}$ & & \\
Day 3 & 1.00 & $<\mathbf{0 . 0 0 1}$ & \\
NNS & 1.00 & $<\mathbf{0 . 0 0 1}$ & 0.75 \\
\hline
\end{tabular}

Table A13: Pairwise post hoc test with Bonferroni adjustment for the ratings of likability between Day 1, Day 2, Day 3, and non-English speaking countries (NNS). The numbers provided in the table are $p$-values for the given pairwise comparison. Ratings provided by workers from non-English speaking countries differ significantly from ratings obtained from workers from English-speaking countries on Day 2. Furthermore, there are significant differences between ratings obtained on Day 1 and Day 2, as well as between ratings obtained on Day 2 and Day 3.

\begin{tabular}{ccccccccc}
\hline & mean (human) & mean (GPT-2) & difference & 95\% CI lower & 95\% CI upper & $\mathrm{t}$ & $\mathrm{df}$ & $p$-val \\
\hline grammar & 4.00 & 3.94 & 0.06 & -0.05 & 0.16 & 1.06 & 1197.9 & 0.29 \\
coherence & 4.11 & 3.82 & 0.29 & 0.18 & 0.42 & 4.97 & 1169.1 & $<\mathbf{0 . 0 0 1}$ \\
relevance & 3.71 & 3.44 & 0.27 & 0.12 & 0.43 & 3.54 & 1184.7 & $<\mathbf{0 . 0 0 1}$ \\
likability & 3.37 & 3.42 & 0.05 & -0.18 & 0.09 & -0.64 & 1194.5 & 0.52 \\
\hline
\end{tabular}

Table A14: Welch's $t$-test on ratings collected on AMT for human-written stories (Day 1) and GPT-2 generated stories. Human-written stories are being rated higher for coherence and more relevance than GPT-2 generated stories $(p<0.05)$.

\begin{tabular}{ccccccccc}
\hline & mean (human) & mean (GPT-2) & difference & 95\% CI lower & 95\% CI upper & $\mathrm{t}$ & $\mathrm{df}$ & $p$-val \\
\hline grammar & 3.86 & 3.94 & 0.08 & -0.19 & 0.03 & -1.50 & 1197.9 & 0.14 \\
coherence & 3.92 & 3.82 & 0.10 & -0.02 & 0.23 & 1.72 & 1176.5 & 0.09 \\
relevance & 3.71 & 3.44 & 0.27 & 0.13 & 0.41 & 3.69 & 1123.5 & $<\mathbf{0 . 0 0 1}$ \\
likability & 3.73 & 3.42 & 0.31 & 0.19 & 0.44 & 4.89 & 1128.9 & $<\mathbf{0 . 0 0 1}$ \\
\hline
\end{tabular}

Table A15: Welch's $t$-test on ratings collected on AMT for human-written stories (Day 2) and GPT-2 generated stories. Human-written stories were rated higher for relevance and likability than GPT-2 generated stories $(p<0.05)$.

\begin{tabular}{ccccccccc}
\hline & mean (human) & mean (GPT-2) & difference & 95\% CI lower & 95\% CI upper & t & df & $p$-val \\
\hline grammar & 3.98 & 3.94 & 0.04 & -0.07 & 0.15 & 0.70 & 1196.4 & 0.48 \\
coherence & 4.05 & 3.82 & 0.23 & 0.12 & 0.36 & 3.98 & 1163.5 & $<\mathbf{0 . 0 0 1}$ \\
relevance & 3.46 & 3.44 & 0.02 & -0.13 & 0.17 & 0.27 & 1188.9 & 0.80 \\
likability & 3.42 & 3.42 & 0.00 & -0.14 & 0.14 & 0.00 & 1192.5 & 1 \\
\hline
\end{tabular}

Table A16: Welch's $t$-test for ratings collected on AMT for human-written stories (Day 3) and GPT-2 generated stories. Human-written stories were rated higher for coherence than GPT-2 generated stories $(p<0.05)$. 


\begin{tabular}{ccccccccc}
\hline & mean (human) & mean (GPT-2) & difference & 95\% CI lower & 95\% CI upper & $\mathrm{t}$ & $\mathrm{df}$ & $p$-val \\
\hline grammar & 3.82 & 3.94 & 0.12 & -0.23 & -0.01 & -2.11 & 1183.1 & $\mathbf{0 . 0 4}$ \\
coherence & 3.45 & 3.82 & 0.37 & -0.49 & -0.23 & -5.42 & 1194.2 & $<\mathbf{0 . 0 0 1}$ \\
relevance & 3.25 & 3.44 & 0.19 & -0.35 & -0.04 & -2.50 & 1185 & $\mathbf{0 . 0 1}$ \\
likability & 3.32 & 3.42 & 0.10 & -0.24 & 0.04 & -1.41 & 1197.9 & 0.16 \\
\hline
\end{tabular}

Table A17: Welch's $t$-test for ratings collected on AMT for human-written stories (non-English speaking countries) and GPT-2 generated stories. GPT-2 generated stories were rated higher for grammar, coherence, and relevance than human-written stories $(p<0.05)$

\begin{tabular}{ccccccccc}
\hline & mean (human) & mean (GPT-2) & difference & 95\% CI lower & 95\% CI upper & $\mathrm{t}$ & $\mathrm{df}$ & $p$-val \\
\hline grammar & 3.83 & 3.82 & 0.01 & -0.09 & 0.12 & 0.28 & 1188.1 & 0.78 \\
coherence & 3.83 & 3.39 & 0.44 & 0.32 & 0.57 & 6.92 & 1198 & $<\mathbf{0 . 0 0 1}$ \\
relevance & 3.49 & 2.70 & 0.79 & 0.65 & 0.93 & 10.85 & 1198 & $<\mathbf{0 . 0 0 1}$ \\
likability & 3.48 & 2.99 & 0.49 & 0.37 & 0.62 & 7.72 & 1195.2 & $<\mathbf{0 . 0 0 1}$ \\
\hline
\end{tabular}

Table A18: Welch's $t$-test for ratings collected on AMT for human-written stories and GPT-2 generated stories (both stories shown in one HIT). GPT-2 generated stories were rated lower for coherence, relevance, and likability than human-written stories $(p<0.05)$ which is in line with the ratings provided by English teachers. 